\title{
Das Palmetum von Santa Cruz de Tenerife
}

\author{
Stephan Scholz
}

\begin{abstract}
In 1996, a palmetum was founded in Santa Cruz (Tenerife) on a former dump. It is open to the public since January 2014. History and most important palms of the park are outlined in the present paper.
\end{abstract}

\section{Zusammenfassung}

Seit 1996 wird in Santa Cruz (Teneriffa) ein Palmetum auf einer ehemaligen Müllhalde aufgebaut. Im Januar 2014 wurde es für die Öffentlichkeit freigegeben. Die Entstehungsgeschicht des Parks sowie seine wichtigsten Palmen werden vorgestellt.

\section{Einleitung}

Die Hauptstadt Teneriffas, im Nordosten der Insel zwischen dem Meer und dem Fuß des Anagagebirges gelegen, bietet dem Besucher viele Sehenswürdigkeiten. Naturkundlern sei das Museo de la Naturaleza y el Hombre empfohlen, ein gut ausgestattetes Naturkundemuseum. Allgemein botanisch Interessierte sollten den schön angelegten Stadtpark Parque García Sanabria besuchen, 1926 eingeweiht und im Jahr 2006 renoviert, der tropische und subtropische Pflanzen aus fünf Kontinenten beher- bergt. Auch Palmen-Enthusiasten kommen auf ihre Kosten: das Palmetum von Santa Cruz, direkt am Meer in der Nähe des modernen Auditoriums gelegen, bietet eine in Europa einmalige Zusammenstellung von Palmen im Freiland und ist ein hervorragendes Beispiel dafür, wie eine hässliche Müllhalde in einen blühenden Garten verwandelt werden kann.

\section{Von der Müllhalde zum Palmenhain}

Anfang der 1970er Jahre, als Santa Cruz in starkem Wachstum begriffen war, wurde begon-

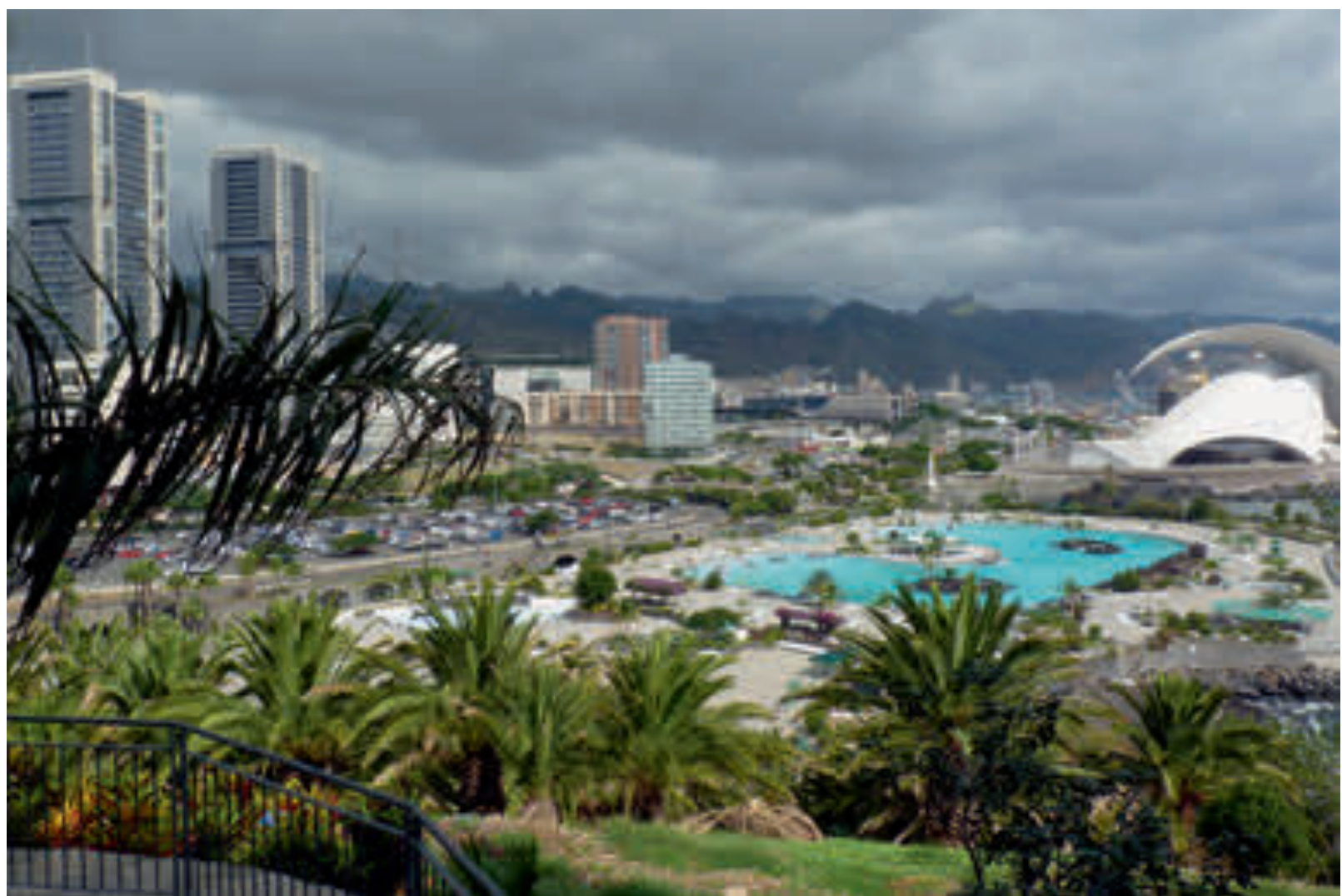


nen, direkt am Meer südlich der Stadt Schutt und Müll abzuladen. Der Berg erreichte schließlich eine Ausdehnung von 12 Hektar und eine Höhe von 40 Metern. Am Anfang lag der Hain wie auch die nicht weit entfernte Erdöl-Raffinerie noch etwas außerhalb der eigentlichen Stadt. Im Zuge des schnellen, immer noch anhaltenden Wachstums von Santa Cruz wurden Raffinerie und Müllberg der Stadt einverleibt und wurden für die in ihrer Nähe lebenden Menschen zu einem Problem.

1983 wurde vom Stadtrat beschlossen, die Müllhalde El Lazareto zu schließen. In den Jahren danach fand eine öffentliche Debatte darüber statt, wie man das Gebiet für die Stadt zurückgewinnen könnte. Schließlich setzte sich die Idee des Agraringenieurs Manuel Caballero durch, hier einen botanischen Garten mit einer umfangreichen Palmensammlung zu errichten.

Im Jahr 1996 begann mit Hilfe von EU-Geldern die Arbeit. Der obere Teil des Müllberges wurde planiert und eine Schicht Erde aufgebracht, die mit Lastwagen aus anderen Teilen der Insel herangebracht wurde. Da im Untergrund durch Gärung große Mengen Methan entstanden, musste ein Gas-Extrahierungssystem installiert werden. Biologen wurden angestellt, die ersten Pflanzen gesetzt und Samen von Palmen aus aller Welt gekauft oder von botanischen Institutionen bezogen. Zu den wichtigsten Arbeiten der nächsten zwei Jahre gehörte der Bau des „Oktogon”, einer halb im Boden versenkten, achteckigen Anlage, in der empfindliche Palmen und andere Pflanzen aus Regenwaldgebieten ein geeignetes, feucht-warmes und windgeschütztes Mikroklima finden sollten. Außerdem wurden mehrere kleine Seen und Teiche angelegt.

Leider folgte nach dieser Anfangsphase ab dem Jahr 2000 eine Zeit der Stagnation: die ursprünglich vorhandenen Gelder waren ver-

Abb. 1 (Seite 142): Blick vom Palmetum auf Santa Cruz.

Abb. 2 (oben): Blühendes Exemplar der seltenen Copernicia ekmanii aus Haiti.

Abb. 3 (unten): Coccothrinax borhidiana ist in ihrer Heimat Kuba eine stark bedrohte Art.
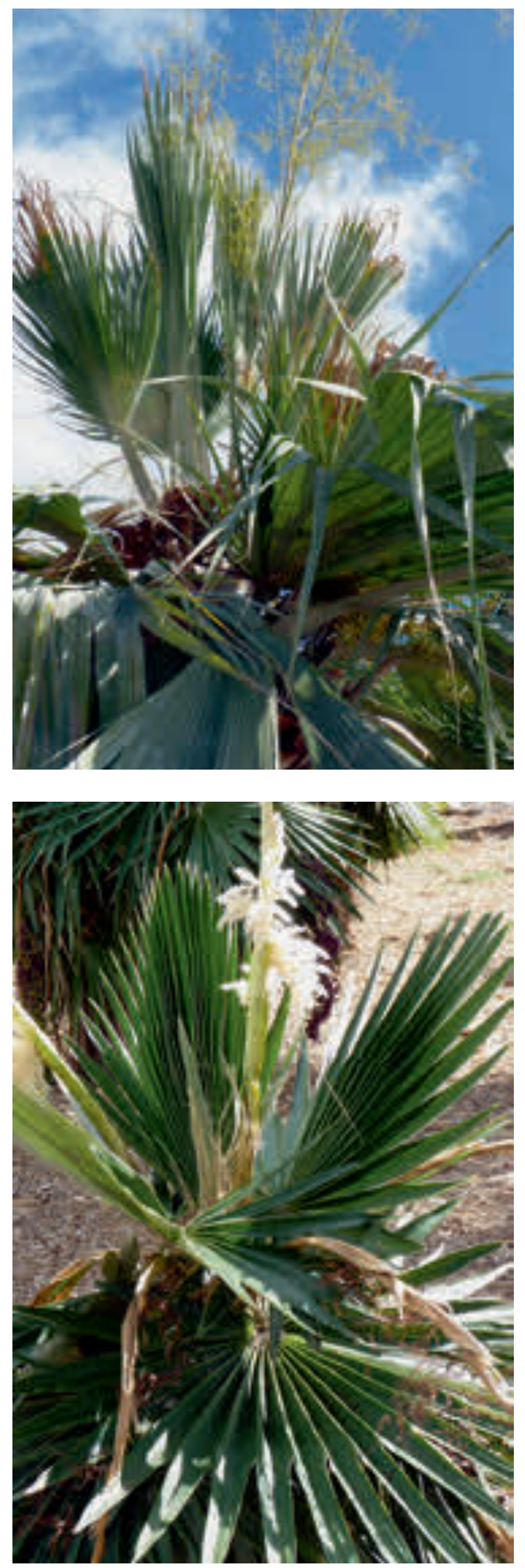


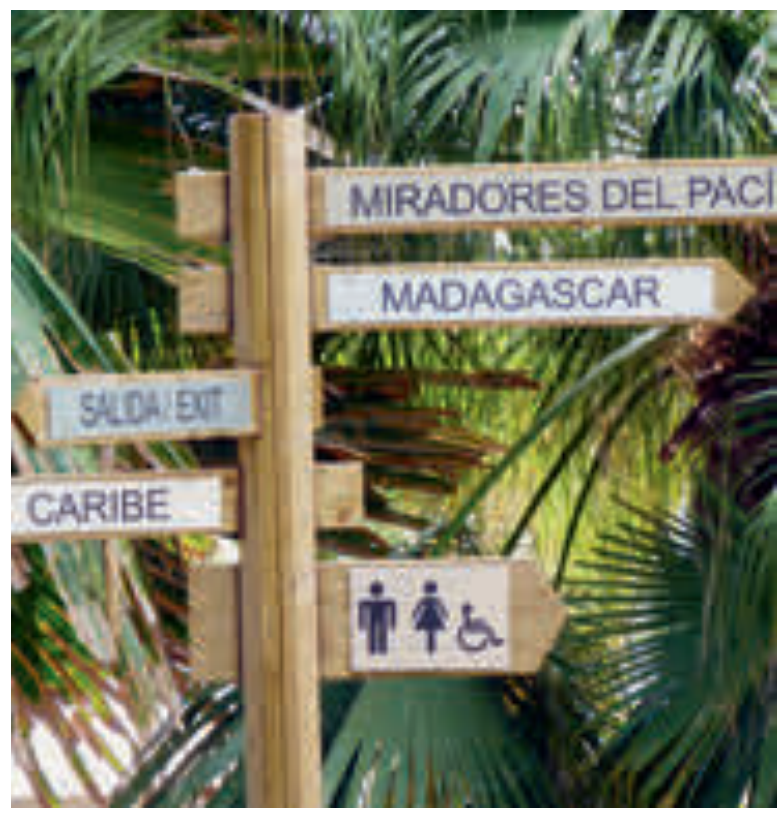

Abb. 4 (oben): Anhand der Wegweiser kann man sich gut im Palmetum orientieren.

Abb. 5 (unten): Teilansicht des Oktogons. Im Vordergrund ist der Korbblütler Ligularia kaempferi zu erkennen. braucht und die Stadt hatte Schwierigkeiten, die Mittel für die korrekte Instandhaltung und den weiteren Ausbau des Palmetums zu beschaffen. Dieses war noch nicht für Besucher zugänglich, weil bis dahin nur etwa ein Drittel der Gesamtfläche bepflanzt und noch kein Wegenetz angelegt war. Trotzdem ging die Entwicklung langsam weiter, hauptsächlich durch den Einsatz und Enthusiasmus derjenigen, die an das Projekt glaubten und versuchten, internationale Aufmerksamkeit und Anerkennung dafür zu gewinnen. Erste Erfolge in dieser Richtung waren die Vorstellung des Palmetums auf dem Kongress der International Biogeographical Society 2004 und der offizielle Besuch des Palmetums Ende des gleichen Jahres durch eine Vertretung der französischen Palmengesellschaft Fous des Palmiers.

Die Kanarische Autonome Regierung begann sich 2007 finanziell an dem Palmetum zu beteiligen. Weitere Teile wurden bepflanzt, unter anderem die steilen Hänge zum Meer. Die

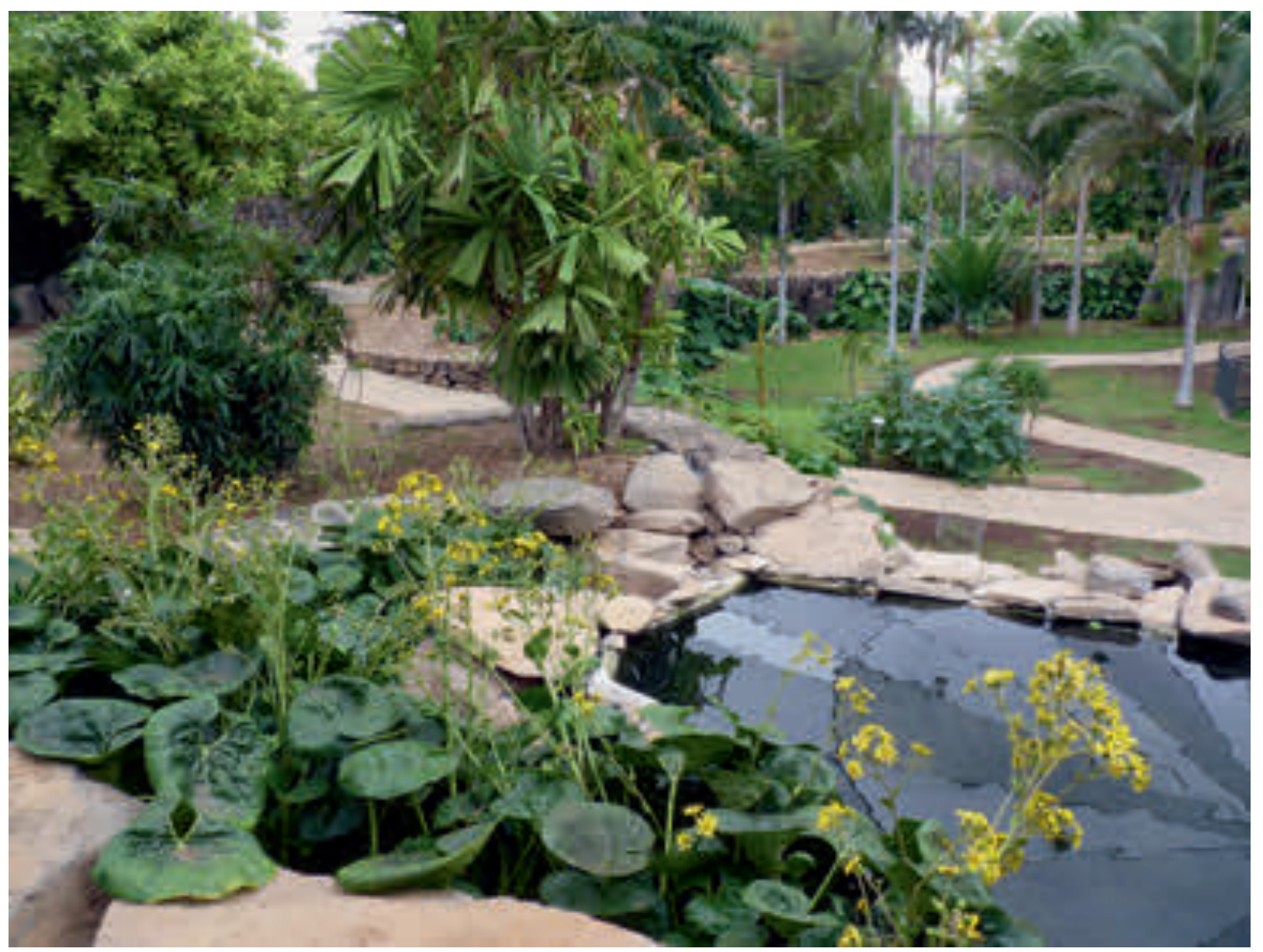


Pflanzensammlungen wurden umfangreicher und die ganze Anlage zum ersten Mal umweltschonend betrieben, in dem auf Pestizide verzichtet und organisches Mulchmaterial ausgebracht wurde.

Nachdem in den Jahren 2010 bis 2013 die letzten Arbeiten wie Beleuchtung, Befestigung der Wege, Anlage von Aussichtspunkten und Beschilderung der Pflanzen ausgeführt wurden, konnte im Januar 2014 die offizielle Eröffnung stattfinden.

\section{Ein Besuch im Palmetum}

Urlauber, die sich in den Touristengebieten im Süden Teneriffas aufhalten (Los Cristianos, Playa de las Américas etc.) erreichen das Palmetum leicht in ca. einer Stunde über die SüdAutobahn. In etwa der gleichen Zeit kann man mit Bus oder Privatauto auch aus Puerto de la Cruz oder anderen Orten im Norden der Insel anreisen. Am nördlichen Fuß des Palmetums befindet sich ein Parkplatz, auf dem der Wagen bequem abgestellt werden kann; kurz dahinter liegt der Eingang. Man kann entweder durch einen Turm mit Wendeltreppe und Fahrstuhl auf dem schnellen Weg direkt in den oberen Teil des Gartens gelangen oder aber den breiten Weg nehmen, der rechts vom Turm langsam den Hang emporführt.

Die Anlage ist nach biogeographischen Regionen gegliedert und beherbergt insgesamt über 400 verschiedene Palmenarten. Um sie im jeweiligen floristischen Kontext zeigen zu können, kommen dazu zahlreiche andere Pflanzen, unter anderem Ficus- und Brotbaum-Arten ( $A r$ tocarpus), Bromelien, Agaven, Schraubenbäume (Pandanus), Nadelbäume der Gattung Araucaria, verschiedene Baobab-Arten (Adansonia) und weniger bekannte, zum Teil seltene Vertreter der Gattung Plumeria (Apocynaceae) aus dem karibischen Raum. Insgesamt sind an die 2000 Arten vertreten. Etwa 100 davon sind

Abb. 6 (oben): Teich mit Mangroven und Typhonodorum lindleyanum.

Abb. 7 (unten): Aechmea blanchetiana im Oktogon vor einem Basaltwasserfall.
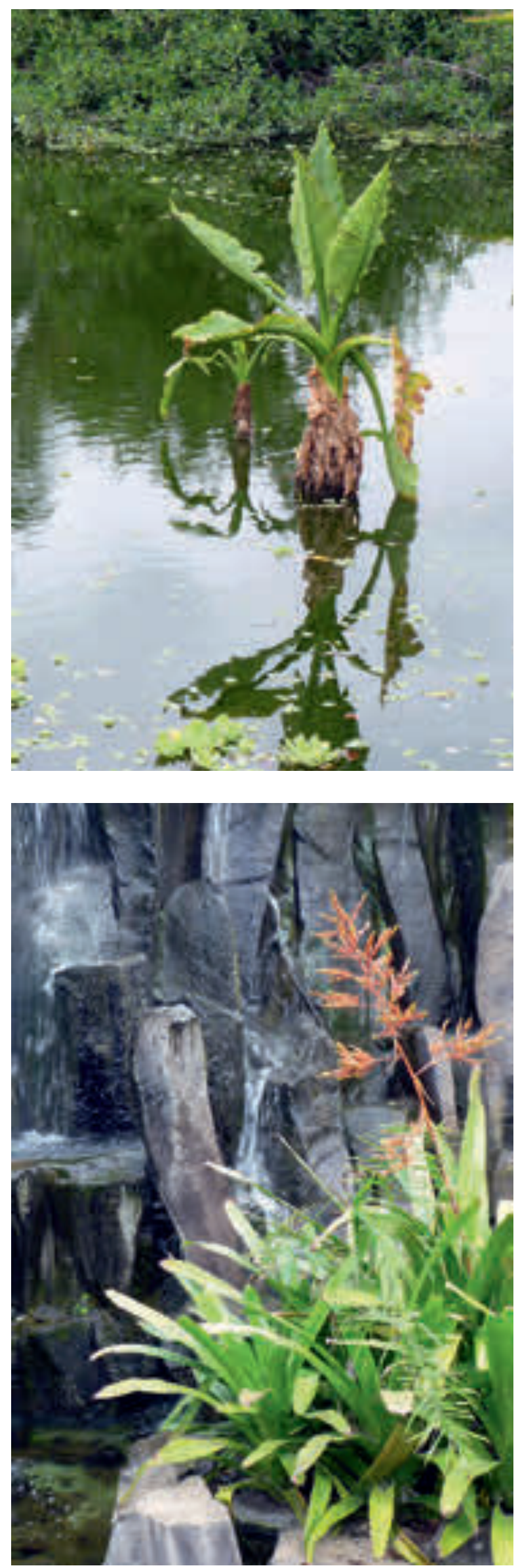


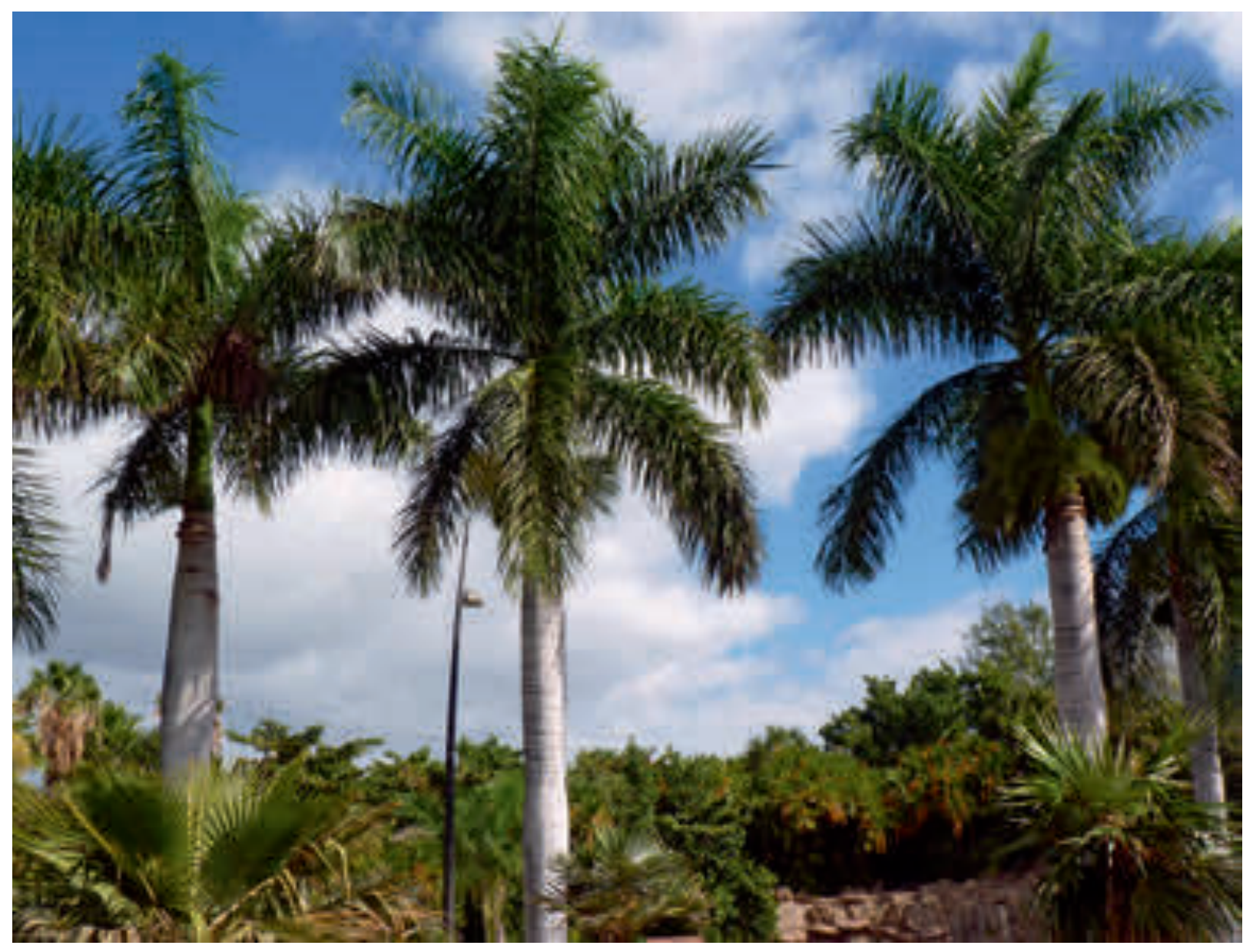

von der IUCN als gefährdet eingestuft und zum Teil sehr selten in Kultur.

Ein etwa $11000 \mathrm{~m}^{2}$ großer Bereich des nördlichen Hanges in der Umgebung des Turms ist vorwiegend mit Kanarischen Dattelpalmen (Phoenix canariensis) bepflanzt. Diese auf den Kanaren endemische Art bildet vor allem auf Gomera und Gran Canaria in mittleren Höhenlagen Wälder mit einer reichen Begleitflora. Der makaronesische Drachenbaum (Dracaena draco) gehört dazu, heute ist er auf unzugängliche Felsstandorte beschränkt. Hinzu kommen Bäume wie Kanarischer Ölbaum (Olea cerasiformis), Wacholder (Juniperus turbinata), Pistacia atlantica, das Lorbeergewächs Apollonias barbujana und die Myrsinacee Heberdenia excelsa. Die beiden Letztgenannten kommen auch in den Lorbeerwaldformationen vor. Im Herbst fallen am Wegrand im kanarischen Bereich des Palmetums blühende Trichter-Narzissen (Pancratium canariense) auf. Sie stammen von den letzten Exemplaren dieser Art ab, die im Stadt- gebiet von Santa Cruz vor der vorrückenden Bebauung gerettet wurden.

Folgt man dem Weg rechts des Turms weiter, kommt man in eine Zone mit verschiedenen Arten der Fächerpalmengattung Pritchardia, die vor allem im pazifischen Raum vertreten ist und besonders auf Hawaii viele bedrohte endemische Arten umfasst. Am Wegrand steht auch ein Exemplar von Colvillea racemosa (Fabaceae). Dieser prachtvolle Baum aus Madagaskar, den man auf den Kanaren sonst kaum antrifft, blühte 2013 zum ersten Mal im Palmetum. Auf der Hochfläche gedeihen der Hawaii-Korallenbaum (Erythrina sandvicensis) und mehrere Noni-Sträucher (Morinda citrifolia), deren Fruchtextrakt heute zunehmend in der alterna-

Abb. 8 (links): Attraktive Gruppe aus bereits recht großen Königspalmen (Roystonea regia).

Abb. 9 (rechts): Blick in das Palmetum mit verschiedenen Palmenarten. 


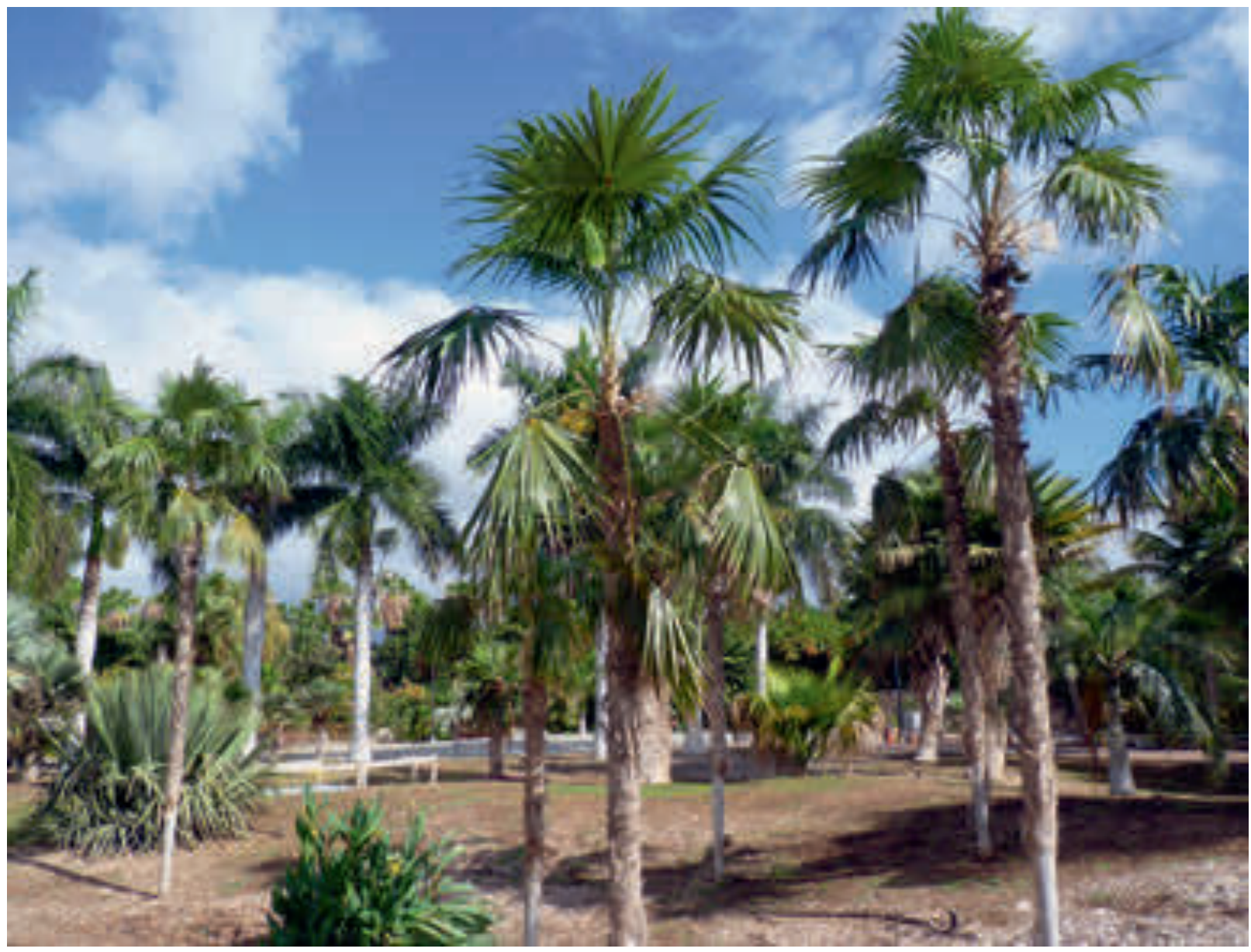

tiven Heilkunde bei bestimmten Arten von Tumoren eingesetzt wird.

Beim Weitergehen in Richtung Osten, zum Meer hin, wird der Madagaskar-Bereich erreicht. Hier wachsen etwa 45 der über $170 \mathrm{Pal}-$ menarten der Insel, von denen alle bis auf 5 endemisch sind (Dransfield \& Beentje 2003). Madagaskar ist somit eines der an Palmenarten reichsten Gebiete der Erde - der gesamte afrikanische Kontinent hat nur ca. 60 Arten aufzuweisen. Selbst in unserer Zeit werden hier noch weitere Palmenarten entdeckt. Dransfield \& Beentje (2003) beispielsweise kannten noch nicht die äußerst seltene, erst im Jahr 2007 in Nordwest-Madagaskar gefundene Fächerpalme Tahina spectabilis. Weniger als 100 ausgewachsene Exemplare dieser Art sind in der Natur bekannt. Sie blüht nur einmal mit einem riesigen, endständigen Blütenstand und stirbt dann ab. Drei junge Exemplare der Art sind im Palmetum zu sehen; bisher wird die Art nur in sehr wenigen botanischen Gärten gezeigt.
Den weitaus größten Teil (über 140 Arten) der Palmen Madagaskars stellt die Gattung Dypsis, in der von sehr großen, die meisten Waldbäume überragenden Arten bis hin zu kleinen, kaum $40 \mathrm{~cm}$ hoch werdenden Palmen aus dem Unterholz alle möglichen Größen vorkommen. Selten in Kultur ist die extrem langsam wachsende D. xerophila aus den Dornbusch-Gebieten im Südwesten der Insel. Viele der Arten sind durch Lebensraum-Verlust stark bedroht, andere wie die Goldfruchtpalme ( $D y p$ sis lutescens) und die Dreieckspalme (Dypsis decaryi) gehören auch auf den Kanaren zu den beliebtesten Zierpalmen. Verschiedene Baobabs (Adansonia-Arten) aus Madagaskar, wo die Gattung mit sechs endemischen Arten vertreten ist, vervollständigen diesen Teil des Gartens.

Im Zentrum der Hochfläche befinden sich mehrere Teiche, deren Ufer mit Mangroven (Rhizophora mangle) bepflanzt sind. Im Wasser, auf dessen Oberfläche sich Wasserhyazinthen (Eichhornia crassipes) und Wasserfarne (Salvi- 


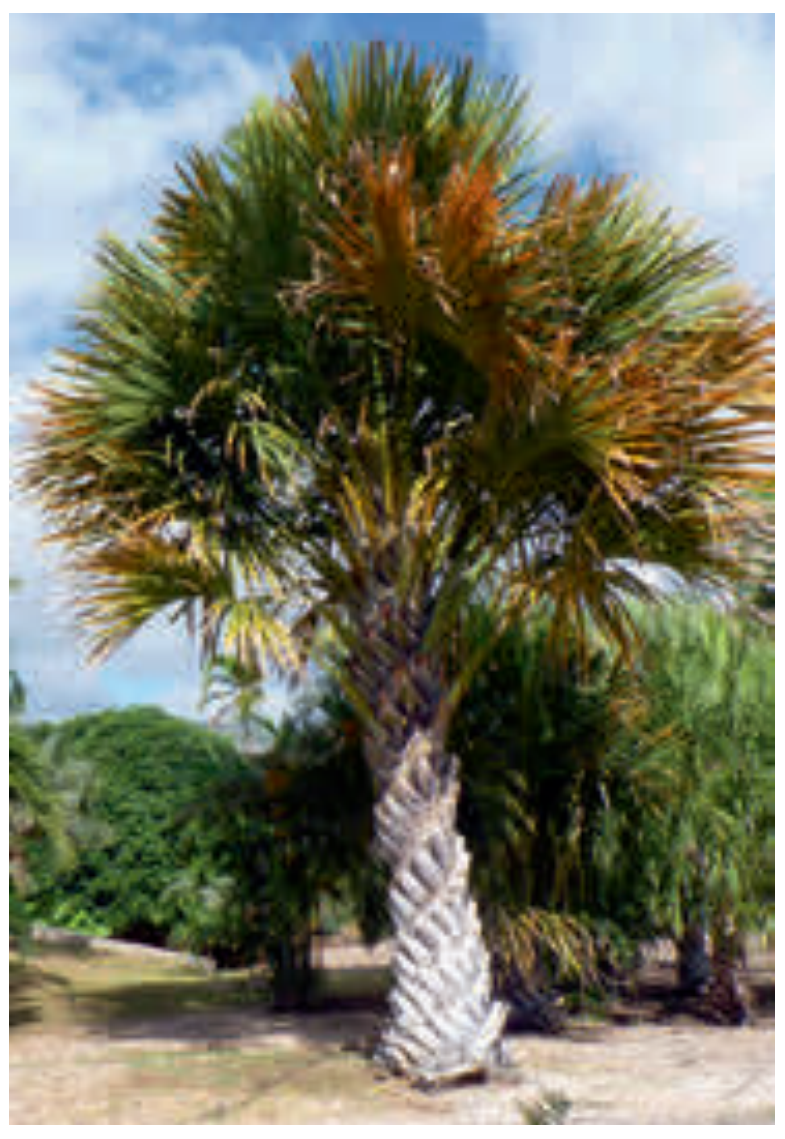

nia) ausbreiten, stehen mehrere Exemplare des großen ostafrikanischen Aronstabgewächses Typhonodorum lindleyanum. Auf den benachbarten Hängen gibt es Gruppen der strauchförmigen Plumeria pudica aus Mittelamerika, Kolumbien und Venezuela. Im Gegensatz zu der häufig in Gärten kultivierten Frangipani (Plumeria-rubra-Hybriden) ist die Art auf den Kanaren noch kaum verbreitet.

Im Asien-Bereich stehen Palmen der Gattungen Arenga, Livistona, Licuala und Corypha beisammen. Die Corypha-Arten, von denen $C$. umbraculifera aus Süd-Indien und Sri Lanka sowie C. utan von den Andaman-Inseln und Nord-Australien im Palmetum zu sehen sind, haben die größten Blütenstände aller Pflanzen; nach der Blüte sterben diese Palmen ab.

Australien und einige umliegende Inselgebiete stellen Palmen der Gattungen Archontophoenix, die auch beliebte Zierpflanzen sind, Arenga, Linospadix, Ptychosperma, Veitchia, Wodyetia und andere. Von der Lord-Howe-Insel östlich Australiens kommen die beiden HoweaArten $H$. forsteriana, die als Kentia weltweit als
Zimmerpflanze verbreitet ist, sowie $H$. belmoreana. Besonders interessant ist die Palmenflora (und auch die übrige Pflanzenwelt) der ebenfalls östlich von Australien gelegenen Inselgruppe Neu-Kaledonien. Hier kommen fast 40 verschiedene Palmenarten vor, unter anderem Vertreter der endemischen Gattungen Aktinokentia, Basselinia, Cyphokentia und Kentiopsis.

In der Karibik-Abteilung sind unter anderem Arten der Gattungen Roystonea, Copernicia und vor allem Coccothrinax im Palmetum vertreten. Die meisten der über 50 Arten dieser Gattung sind auf Kuba endemisch und dort zum Teil sehr selten, so zum Beispiel C. borhidiana, die nur auf Kalkstein in einem eng begrenzten Gebiet an der Nordwestküste der Insel heimisch ist. Eine Gruppe von mehr als 20, regelmäßig fruchtenden Exemplaren ist im Palmetum zu sehen. Ebenso selten ist die nur in Haiti vorkommende Copernicia ekmanii. Zu sehen sind auch die kubanischen Hemithrinax compacta, die auf Serpentingestein vorkommt, und die als kritisch gefährdet eingestufte Hemithrinax ekmaniana, die nur in einem sehr kleinen Karstgebiet lebt. Häufiger und als Zierpalme in allen tropischen Ländern verbreitet ist die Königspalme (Roystonea regia).

Besonders schön gestaltet ist das Oktogon im östlichen Teil der Ebene im oberen Teil. An seiner Innenwand fällt hier Wasser ein paar Meter über Basaltstufen und bildet auf dem Grund kleine Teiche. Das Spritzwasser trägt zur Erhöhung der Luftfeuchtigkeit bei. Das Oktogon ist je etwa zur Hälfte mit Palmen aus den tropischen Wäldern der Alten und der Neuen Welt bepflanzt. Es sind Arenga undulatifolia aus Südostasien und verschiedene Arten aus der Gattung Licuala vertreten, auch Kletterpalmen der Gattung Desmoncus, die aus den Wäldern Mittel- und Südamerikas stammen, sowie die schöne, fast ungeteiltblättrige Pelagodoxa henryana

Abb. 10 (oben): Corypha utan.

Abb. 11 (Seite 149 oben): Im Palmetum gedeihen nicht nur Palmen. Blüte der Kanarischen Trichter-Narzisse.

Abb. 12 (Seite 149 unten): Blütenstand von Colvillea racemosa. 
von den Marquesas-Inseln im tropischen Pazifik. Dazu kommen viele Aronstabgewächse (Anthurium, Phylodendron, Alocasia u.a.) und Bromelien, darunter die großwüchsige, robuste Aechmea blanchetiana aus Brasilien. Hier findet man auch ein Exemplar von Ficus aspera var. parcelii mit unregelmäßig weiß panaschierten, rauen Blättern.

Östlich des Okotogons erstreckt sich ein Bereich, der den afrikanischen Palmen gewidmet ist. Hier stehen Arten der Gattungen Borassus und Raphia, die weltweit in den Tropen angebaute Ölpalme (Elaeis guineensis) und die südafrikanische Jubaeopsis caffra. Es gibt auch einige junge Exemplare der seltenen, lange Zeit verschollenen Fächerpalme Medemia argun aus den Wüstengebieten Ägyptens und des Sudan, die 1995 wiederentdeckt wurde (GibBons \& Spanner 1996, 1999). Ihre Samen wurden zuerst in altägyptischen Gräbern gefunden. Die Palme ist mit den Arten der Gattung Hyphaene sowie mit Bismarckia nobilis aus Madagaskar verwandt und gilt als kritisch vom Aussterben bedroht.

Vom Palmetum schweift der Blick über das Meer und die Stadt Santa Cruz. Dieser Palmengarten wird in Zukunft weiter ausgebaut, wobei vor allem die Hangbereiche zum Meer mit Palmen bepflanzt werden sollen, die bis jetzt nur mit Bodendeckern wie Carpobrotus und verbreiteten Gartenpflanzen wie Acalypha bedeckt und für das Publikum noch nicht zugänglich sind. Geplant ist auch ein Palmen-Museum; ferner sollen Vogel-Beobachtungspunkte eingerichtet werden. Man kann aber schon jetzt nur empfehlen, das Palmetum in Santa Cruz bei einem Besuch auf jeden Fall mit einzuplanen.

\section{Literatur}

Dransfield, J. \& Beentje, H. 2003: Arecaceae, Palms. In: S. M. Goodman \& J. P. Benstead (Hrsg.):

The natural history of Madagascar. - Chicago, London. Gibbons, M. \& Spanner, T. W. 1996: Medemia argun lives. - Principes 40: 65-74.

Gibbons, M. \& Spanner, T. W. 1999: In the valleys of the Sudan: finding Medemia. . - Palm J. 149: 33-35.

Lötschert, W. 1985: Palmen. - Stuttgart.

\section{Internetseite}

http://palmetumtenerife.es/
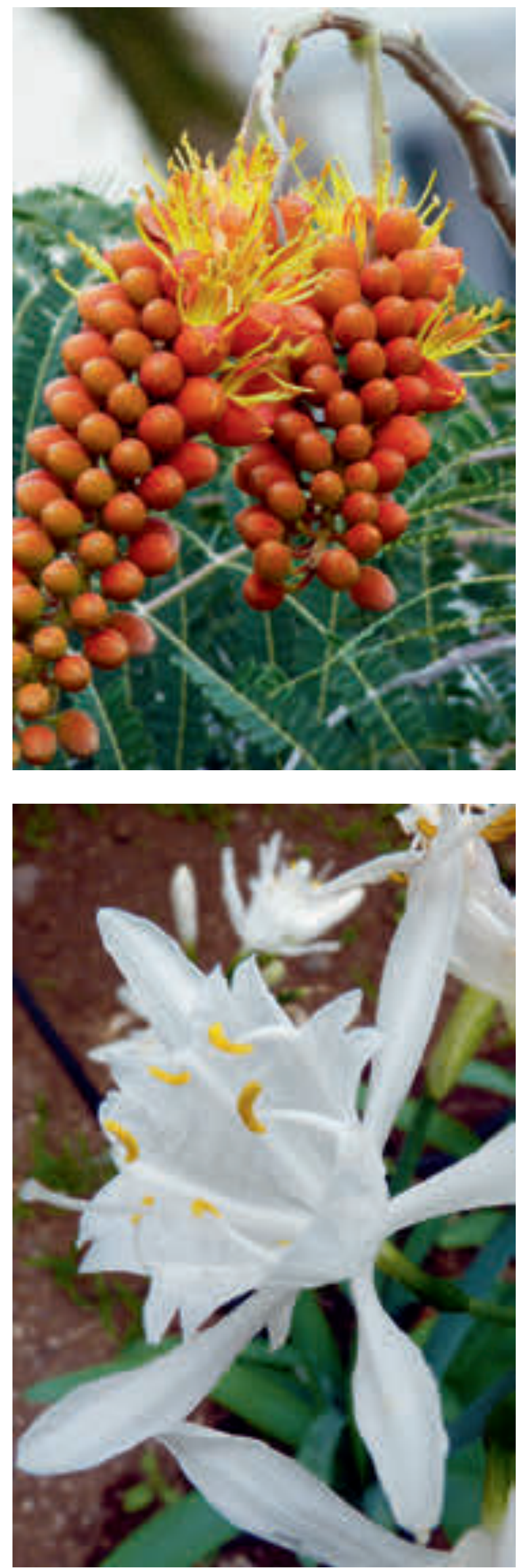